\title{
Detection of Subsurface Defects in Steels using Pulsed Eddy Current Thermography
}

\author{
by A. Taram***, C. Roquelet*, M. Anderhuber* P. Meilland*, K. Mouhoubi**, JL Bodnar**
}

*ArcelorMittal Global Research and Development, France, abdoulaye.taram@arcelormittal.com, cyrielle.roquelet@arcelormittal.com, philip.meilland@arcelormittal.com

**University of REIMS, GRESPI/CATHERM, France, jl.bodnar@univ-reims.fr, kamel.mouhoubi@univ-reims.fr

\begin{abstract}
Eddy current thermography [1-5] consists in using electromagnetic induction to create heat flow in the material and record its thermal response using an infrared camera. Defect detection is based on the changes of the thermal distribution. It is an efficient and precise method for detecting subsurface defect in metallic materials and can be deployed either in the form of pulses or amplitude modulations [3, 4]. This paper describes simulation works using Pulsed Eddy Current Thermography for defect detection and quantification in material made of steel in transmission mode. Flux2D Finite Element Software is used
\end{abstract}

Key words: Defect detection, Defect quantification, Eddy Current thermography, heat diffusion

\section{Introduction}

Pulsed Eddy Current Thermography (PECT) consists in generating a heat pulse with Eddy-currents, which will propagate in the material; a defect will hamper locally the propagation of this heat wave, and will be captured by an infrared camera and evaluated. This technique merges advantages of Eddy Current NDT and outstanding merit of pulsed thermography i.e. low time consuming. Furthermore, camera which is the main instrument for performing infrared thermography has been improved in both, sensibility and in spatial resolution. In addition to that, the computational resources required for control, data acquisition, storage and processing have been updated, providing thus more computing power to perform complex processing tasks. These factors have made PECT more attractive for subsurface defect detection and quantification in metallic components and structures. [5-8]. Defect quantification is of interest so that to judge its severity. In general, defect characterization is performed from temporal response or spatial response. Most of the works based on defect quantification, is based on the thermal contrast and phase data analysis under reflection mode. For example, a relationship was established between the thermal maximum contrast $C_{\text {max }}$ and the time at which it appears $t_{\text {max }}$ in order to retrieve defect depth [9]. Maldague and Ibarra-Castenado proposed to retrieve the defect depth by using phase data. We are aiming in this paper, to use thermal contrast to quantify defects in transmission mode. Vageswar et al. have investigated quantification method for wall thinning defect under transmission mode [10].

In transmission mode, the camera records the temperature evolution of the rear face of the sample whereas in reflection mode, the camera records the temperature evolution of the front face where the heating source is applied (Figure 1).This paper is focused in subsurface defect detection and quantification using transmission mode. Simulations will be used to allow better comprehension of fundamental phenomena surrounding defect detection with Pulsed Eddy Current Thermography. 


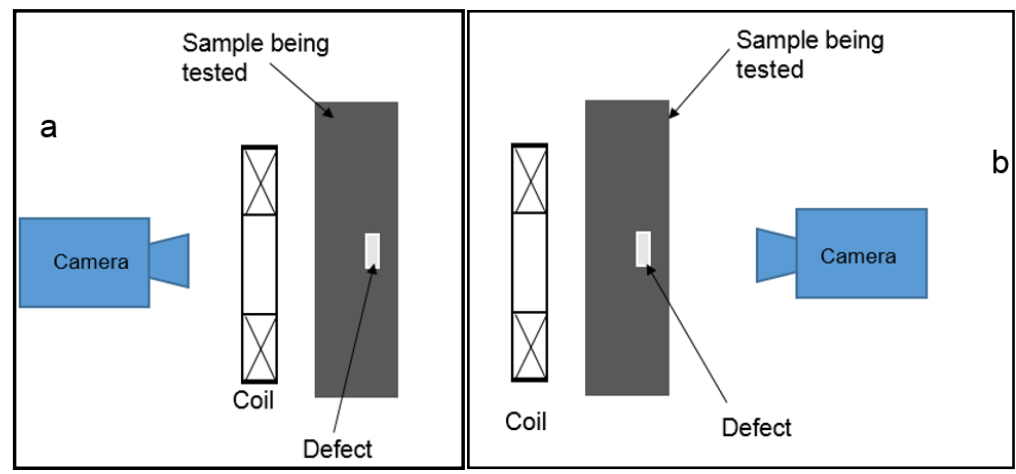

Figure 1: Schematization of the two modes (a: Observation of front face temperature response; $b$ : Observation of rear face temperature response)

\section{Fundamentals of Pulsed Eddy Current Thermography}

PECT is based on the application of a short period and high frequency current. The induced Eddy currents are converted to heat through ohmic or resistive heating, according to Joule's Law. The surface temperature of the material first rises during the application of the pulse then decays as the thermal wave propagates by diffusion through the material. Then the surface temperature is the contribution from direct Eddy current heating and diffused heat. However, the pulsed Eddy current has a penetration depth known as skin depth which is given by the relationship below:

$$
\delta=\frac{1}{\sqrt{\pi \mu \sigma f}}
$$

Where $\delta$ is the skin effect [m], $\mu$ the magnetic permeability $(\mathrm{H} / \mathrm{m}), \sigma$ the electrical conductivity $(\mathrm{S} / \mathrm{m})$ and $f$ the excitation frequency $(\mathrm{Hz})$.

Meanwhile the ohmic heating $Q$ is proportional to the resistivity $\left(\frac{1}{\sigma}\right)$ of the material and the square of the Eddy current density, $J$.

$$
Q=\frac{1}{\sigma} J^{2}=\delta^{2} \pi \mu f J^{2}
$$

The generated ohmic heating depends on the type of materials and the frequency of excitation. The presence of a subsurface defect will reduce the diffusion rate, leading to a heat accumulation around the defect and consequently an area of higher temperature at the material surface with respect to the surrounding material. The defects which are located in the range of the Eddy current penetration depth will disturb the current flow and thus change the temperature distribution. Hereby, direct interaction with Eddy current improves the near-surface defect detection. The defects which do not directly interact with the induced Eddy currents will interact with the heat flow spreading. In this paper, we are studying the temperature response of the rear face. Then the detection of the defect will result from the interaction between the diffused heat and the defect. In absence of defect, the 1D solution of the heat diffusion equation for transmission mode is given by [12-13]:

$$
T(t)=\frac{Q}{\rho C_{p} L}\left[1+2 \sum_{n=1}^{\infty}(-1)^{n} \exp \left(-\frac{n^{2} \pi^{2}}{L^{2}} \alpha t\right)\right]
$$

Where $Q$ is heat supplied to the sample surface, $\rho$ is density, $C_{p}$ is specific heat, $L$ is the thickness of sample, $\alpha=\frac{k}{\rho C_{p}}$ is the thermal diffusivity, and $k$ the thermal conductivity. 


\subsection{1/qirt.2016.133}

\section{Defect detection}

In rear face observation, IR Camera, placed opposite to the heating source (as shown in Figure 2), records the thermal response. The analysis of this response leads to the detection of the defect. The defect detection principle is based on temperature differences. Maldague [9] states that defect can easily be detected if width-to-position-in- thickness ratio $>2$. By respecting this condition we likely avoid the loss of defect visibility as defect visibility is based on thermal contrast.

Fourier number which is used to characterizes transient heat conduction; establish the relationship between the thermal diffusion and the thermal energy storage. It's designated by $F_{0}$ and given by [9] :

$$
F_{0}=\frac{\alpha t}{L^{2}}
$$

Here $\alpha$ is the thermal diffusivity $\left(\mathrm{m}^{2} / \mathrm{s}\right), \mathrm{t}$ is the time $(\mathrm{s})$, and $\mathrm{L}$ is the thickness of conditional layer $(\mathrm{m})$.

By considering the 3D heat diffusion problem, the Fourier number for each direction is written as [14]:

$$
F_{0 x}=\frac{\alpha_{x} t}{L_{x}{ }^{2}} \quad F_{0 y}=\frac{\alpha_{y} t}{L_{y}{ }^{2}} \quad F_{0 z}=\frac{\alpha_{z} t}{Z^{2}}
$$

Where $\alpha_{x}, \alpha_{y}, \alpha_{z}$ represent the diffusivity, $L$ the lateral size, and $Z$ the defect position in thickness regard with front surface.

In reflection mode, the thermal contrast degradation takes place when $F_{0 x}$ and $F_{0 y}$ are predominant i.e. $F_{0 x}, F_{0 y}>>F_{0 z}$. Consequently defects with smaller width-to-position-in-thickness ratio will need special data processing to be detected. The heat transfer in the defect area during the transient period can be considered one dimensional when the lateral size of the defect is large and the heat conduction through the air gap is negligible.

\section{Simulation}

The aim of this part is to understand the involved phenomenology of defect visibility and to study its applicability. A very thick steel sample was modeled with Flux2D Finite Element method software. Two temperatures sensors are placed respectively in front of defect area and defect free area. The defect region is filled with air. The front surface of the sample is heated by a coil and the sensors are used to record the rear face temperature response.

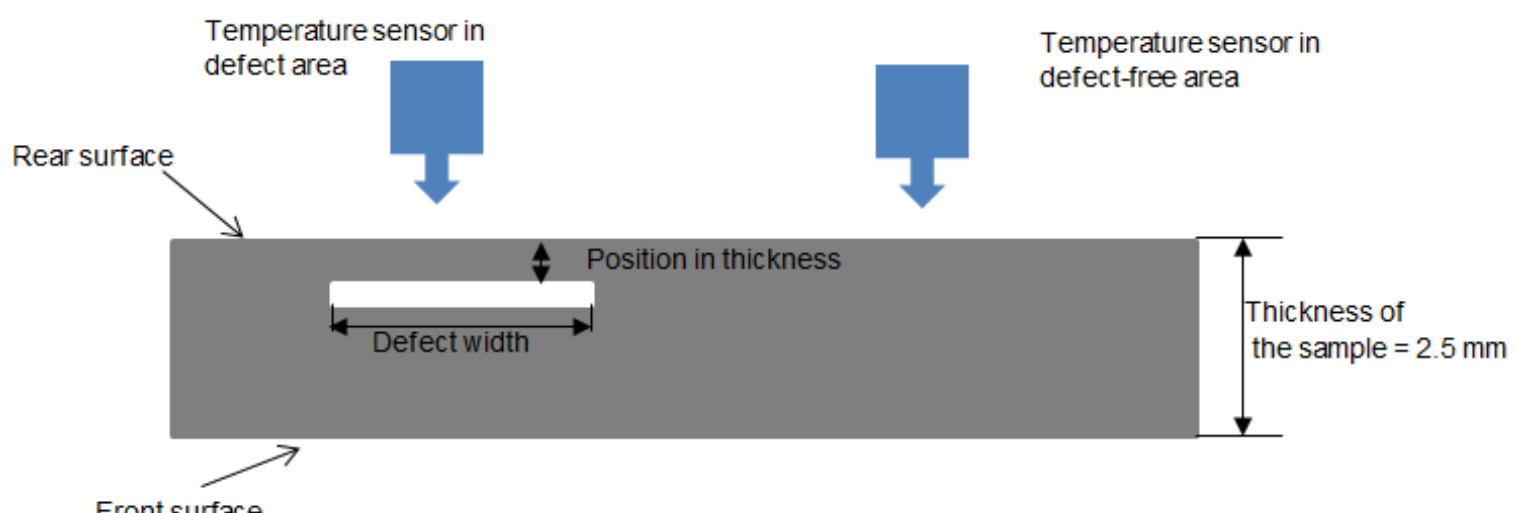

Front surface

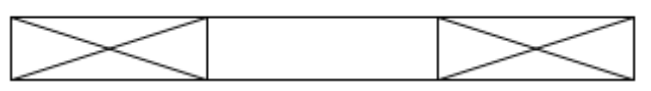

Coil

Figure 2 : Geometric configuration 
The properties of the sample and defective region are mentioned in the table below.

\begin{tabular}{|c|c|c|}
\hline Properties & Steel & Air \\
\hline Density $\left[\mathrm{kg} / \mathrm{m}^{3}\right]$ & 7800 & 1.3 \\
\hline Heat capacity $[\mathrm{J} /(\mathrm{kg} . \mathrm{K})]$ & 500 & 1010 \\
\hline Thermal conductivity $[\mathrm{W} / \mathrm{m} . \mathrm{K})]$ & 50 & 0.026 \\
\hline Electrical conductivity $[\mathrm{S} / \mathrm{m}]$ & $1.10^{6}$ & 0 \\
\hline Relative permeability & 100 & 1 \\
\hline
\end{tabular}

Table 1: Thermo physical properties of the sample and air

A series of simulation was launch so that to figure out the effect of defect lateral size and position within the sample on defect visibility. Fernando et Al. have already performed this analysis in reflection mode [14]. In this article, we are highlighting the effect on rear face temperature response. Then, during simulation, the excitation current is set at $10 \mathrm{~A}$ with $200 \mathrm{kHz}$ and the excitation time is set at $30 \mathrm{~ms}$.

\section{Effect of Lateral size on defect visibility}

Firstly, the effect of the lateral size on defect visibility is observed. The thickness of the defect is set at $0.2 \mathrm{~mm}$ (remain constant in this presented work) and its position in the thickness of the sample is set at $0.1 \mathrm{~mm}$ regard with rear face (see Figure 2). With defect width $0.2,0.5,1.5,2.5$ and $4 \mathrm{~mm}$, we have respectively 2, 5, 15, 25 and 40 width-in-thickness ratio.

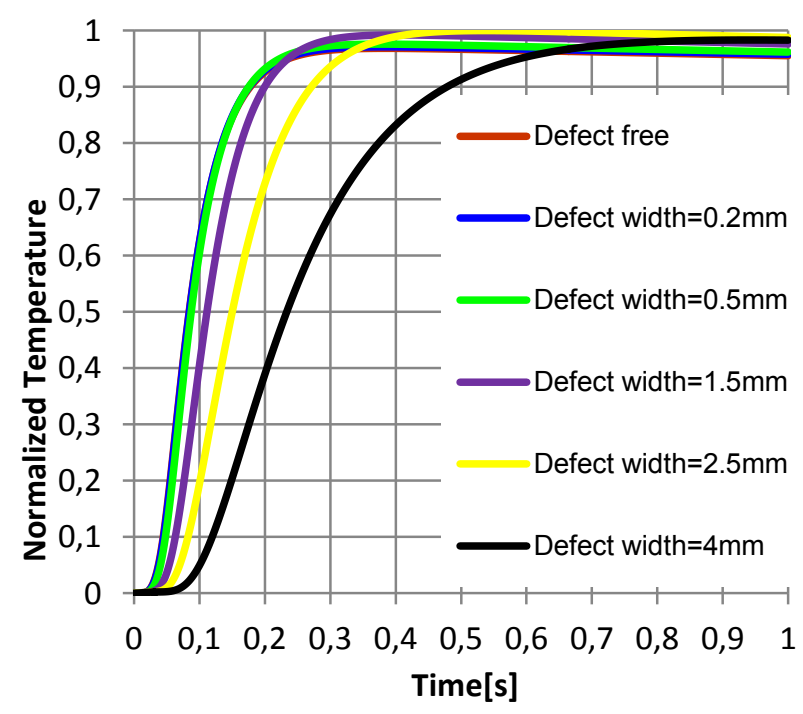

Figure 3 : Normalized transient temperature responses of the rear face for different width of the defect (Defect position in thickness of the sample $=0.1 \mathrm{~mm}$ )

The figure 3 above shows different behavior of the temperature related to the defect width. The generated heat is transferred from the heated surface to the interior of the sample because of heat conduction, and leads to a continuous increase of the temperature of the rear surface. In the subsurface defect area which is filled by air, the heat flux copes with a higher thermal resistance which reduces the heat transfer rate. Consequently, the rear temperature response of defect area is smaller than the defect free area.

Defect is detected when abnormal behavior appears regard with the defect free temperature. Then defect will be displayed as cool (or dark) spot. The Figure 3 illustrates that at the same position in the thickness of the sample, the wider the defect, the better the defect can be detected. In contrary, temperature evolutions of defects whose width $<0.5$ $\mathrm{mm}$ are superposed with the defect free one. Consequently it is difficult to identify the defects whose width $\leq 0.5 \mathrm{~mm}$. 


\subsection{1/qirt.2016.133}

Mainly thermal contrast can be used as the relevant parameter for defect detection and characterization [9]. As seen in the literature, different definitions of thermal contrast are used. In this paper we used the absolute thermal contrast given by the following equation:

$$
\Delta T=T_{s}-T_{d e f}
$$

$T_{d e f}$ refers to the temperature of the defected region, $T_{s}$ to sane region.

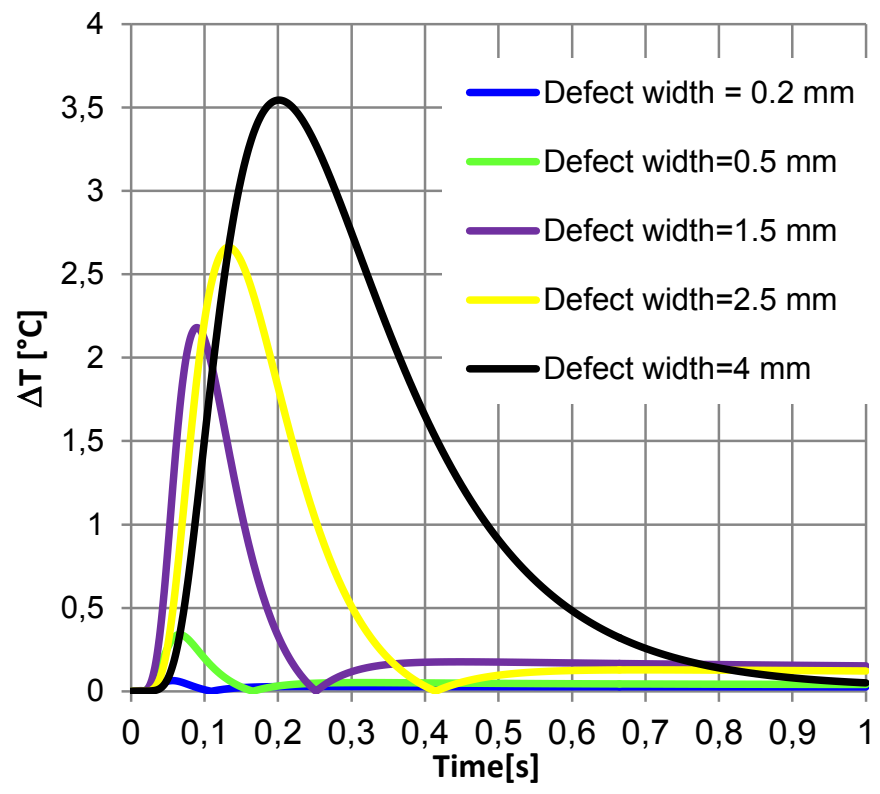

Figure 4: Thermal contrast of the rear face of the sample for different width of the defect (Defect position in thickness of the sample $=0.1 \mathrm{~mm}$ )

Undoubtedly, the wider the defect, the higher the contrast and the better the defect is visible. Absolute contrast computations help enhance defect visibility. Defect width impacts considerably the maximum value of thermal contrast $C_{\max }$ and the time $\left(t_{\max }\right)$ at which it appears.

According to the relationship given by Fourier number, when the lateral size of the defect increases $F_{0 x}$ and $F_{0 y}$ can be neglected regard with $F_{0 z}$. Then $t_{\max }$ shouldn't depend on the lateral size. Since thermal waves will travel the same distance whether their strength is reduced by the presence of a defect or not [9], the 3D heat diffusion phenomenology have less influence on the temperature response of the rear surface.

\section{Effect of defect position in the thickness of the sample on its visibility}

After studying the effect of the lateral size on defect visibility, below we have studied the effect of defect position in thickness within the sample. As illustrated in Figure 2, the defect width is set at $1 \mathrm{~mm}$ and the position in thickness of the defect within the sample is varying. The transient temperature responses at the center point for different positions in thickness of the defects are shown in Figure 5. 


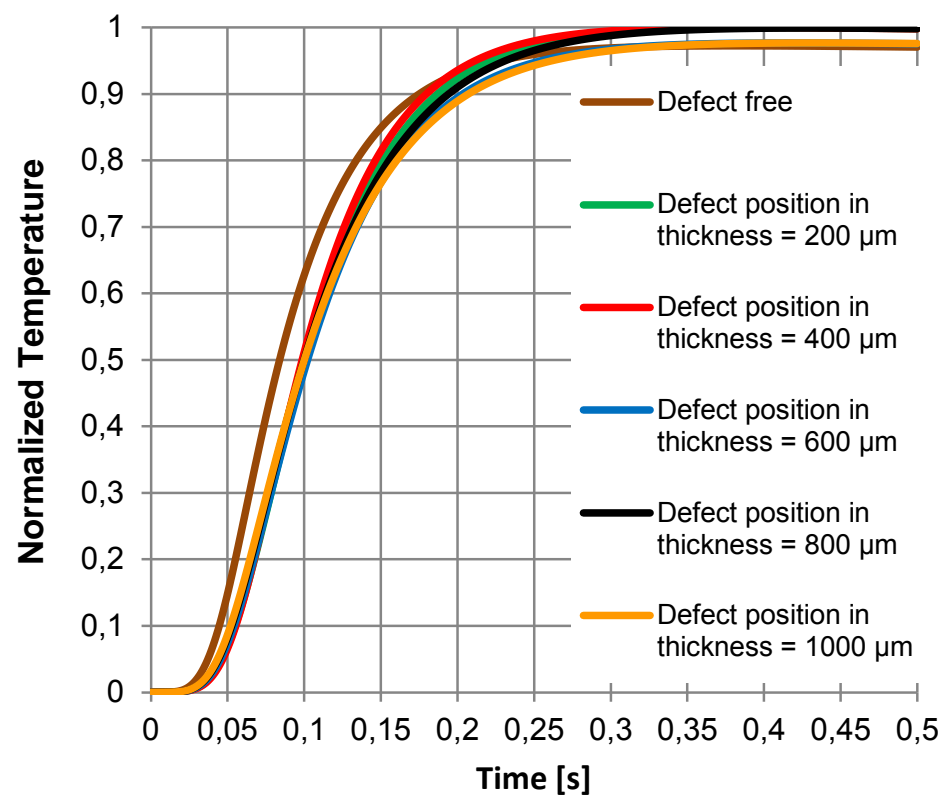

Figure 5 : Normalized transient temperature responses of rear face for different positions of the defect within the sample (defect width $=1 \mathrm{~mm}$ )

In contrary to the Figure 3, it is difficult to observe the influence of defect position in thickness on defect visibility. Nevertheless the defect free curve has different behavior regard with defective curves. As previously done, the thermal contrast calculated to highlight the effect of position in thickness (see Figure 6 ).

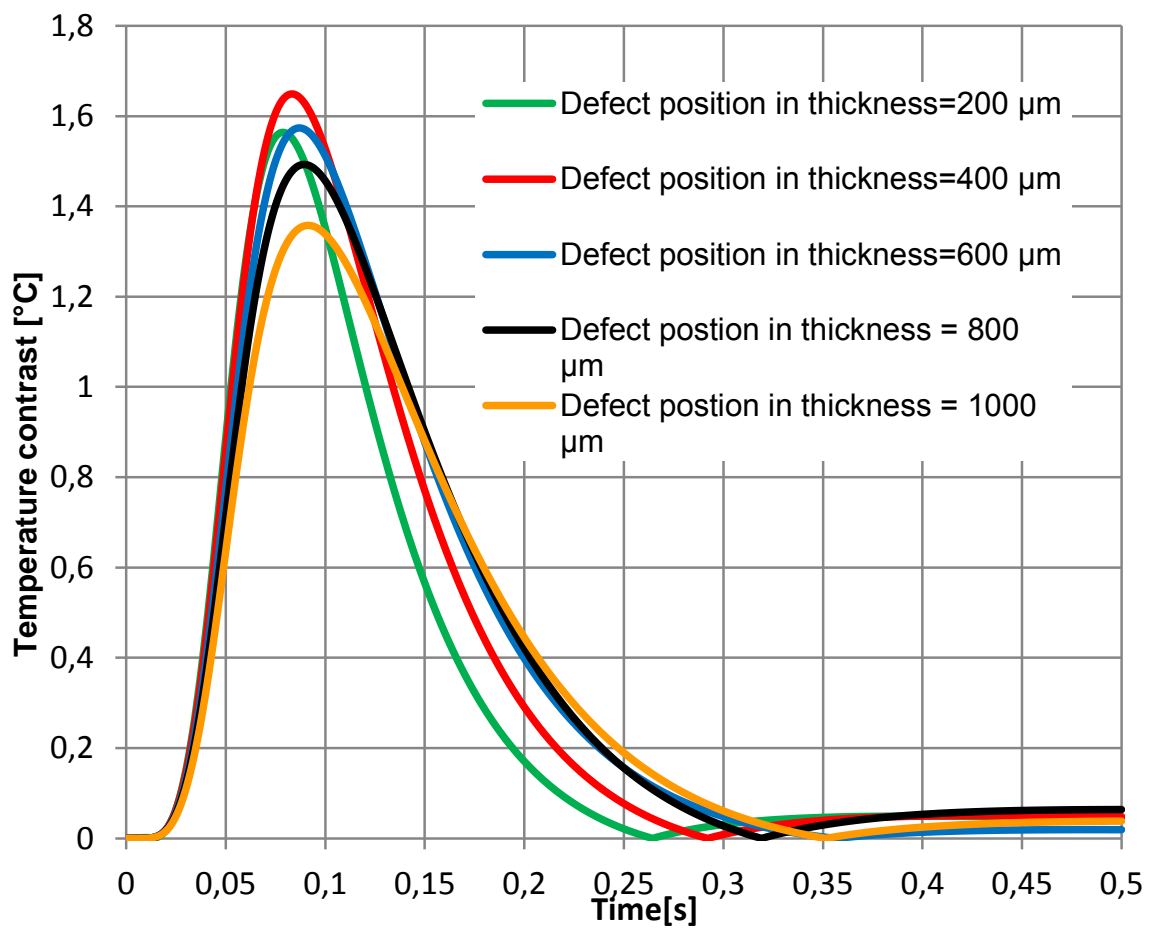

Figure 6 : Temperature contrast of different positions in thickness of the defect (defect width $=1 \mathrm{~mm}$ )

The Figure 6 shows the maximum peak contrast, $C_{\max }$, for different positions in thickness of the defect and the time, $t_{\max }$, at which the maximum contrast appears. To better illustrate the variation, the figure 7 displays $C_{\max }$ and $t_{\max }$ in function of the position in thickness of the defect. One can see that when the defect position in thickness $<400 \mu$ m, the maximum 
contrast increases with the position in thickness. Meanwhile, from position in thickness $=400 \mu \mathrm{m}$, the maximum contrast decreases when the defect position in thickness is increasing as illustrated in figure $7 \mathrm{a}$. We can see that the thermal wave reaches its maximal value at position in thickness is $400 \mu \mathrm{m}$, which corresponds to a relative position $16 \%$ of the thickness of the sample. For defect situated at position higher than $400 \mu \mathrm{m}$, the travelling distance of the transmitted wave higher leading to the reduction of the contrast.

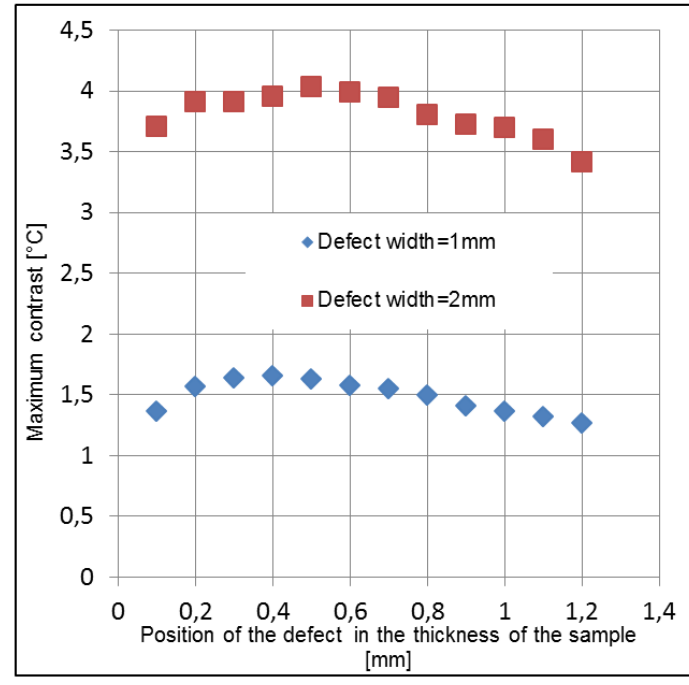

Figure 7 a

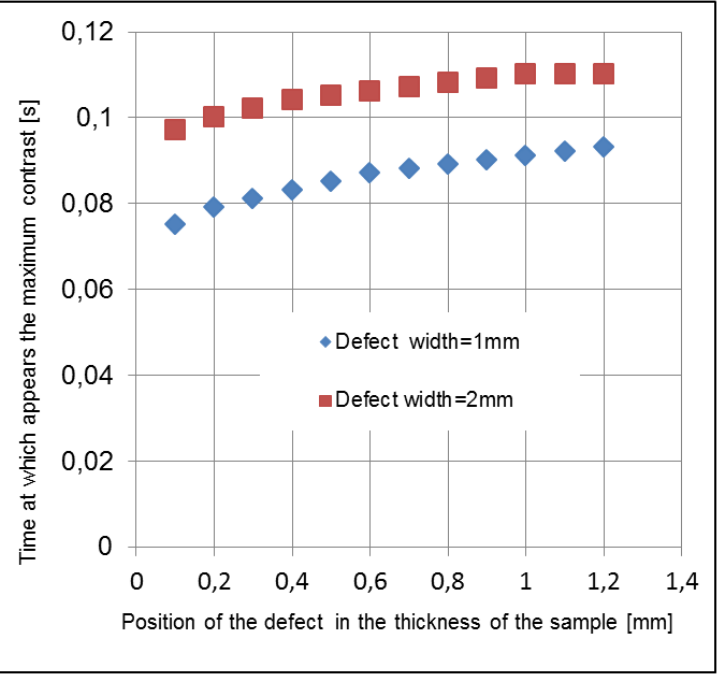

Figure $7 \mathrm{~b}$

Figure 7: Influence of defect position within the thickness on the maximum contrast and its time of occurrence

The figure $7 \mathrm{~b}$ shows the time at which appears the maximum contrast $\left(t_{\max }\right)$. One can see that the relationship between $t_{\text {max }}$ and position in thickness of the defect increases. In other words, deeper defects take longer to be detected dependently on their lateral size. The evolution of $t_{\max }$ appears to be linear for position in thickness greater than 400 $\mu \mathrm{m}$. Hopefully, this relationship could be used to retrieve the position in thickness of the defect. In literature, so far there is no analytical solution to do that in transmission mode. But, in reflection mode, Balageas et al. found that the maximal contrast and time, at which it appears, are linked by [9];

$$
z=A \sqrt{t_{\max }}\left(C_{\max }\right)^{n}
$$

Where $A$ and $n$ are calibration coefficient which depend on the defect width

According to figure $7 \mathrm{a}$ and figure $7 \mathrm{~b}$, the maximum temperature contrast $\left(C_{\max }\right)$ and its time $\left(t_{\max }\right)$ of occurrence are function of both, the position in thickness and width of the defects. This will constitute a constraint in the practical applicability because it will be necessary to know the defect width before estimating its position in thickness.

\section{Conclusion}

In this paper defect detection and characterization based on heat diffusion using pulsed Eddy current thermography are studied. Simulation works provided understandings of the fundamental information on how defect size and location within the sample thickness can influence the defect detection and characterization. The study was done by observing the transient temperature response of the rear face. The absolute contrast computations help to enhance the defect visibility since the thermal contrast is a relevant parameter for defect detection. The absolute contrast values provided by the simulation works are ranging from $1.2{ }^{\circ} \mathrm{C}$ to $3.5^{\circ} \mathrm{C}$. Given that the thermal sensitivity of current IR camera at $25{ }^{\circ} \mathrm{C}$ is in the order of $20 \mathrm{mK}$, this simulation work shows that all the simulated defects presented in this paper should be detectable. In the next step of our study, we will confront those simulations results to experimental measurement on artificially manufactured flaws. 


\section{REFERENCES}

[1] I. Z. Abidin, M. Z. Umar, M. Yusof, M. Ibrahim, A. Hamzah, M. Salleh, « Advantages and Applications of Eddy Current Thermography Testing for Comprehensive and Reliable Defect Assessment ", in Proceedings of 18th World Conference on Nondestructive Testing, Durban, South Africa, 2012, p. 16-20.

[2] B. Oswald-Tranta, G. Walle, J. Oswald, « A semi-analytical model for the temperature distribution of thermo inductive heating », QIRT Padova Italy, 2006.

[3] C. Ibarra-Castanedo, M. Genest, J.-M. Piau, S. Guibert, A. Bendada, X. P. V. Maldague, « ACTIVE INFRARED THERMOGRAPHY TECHNIQUES FOR THE NONDESTRUCTIVE TESTING OF MATERIALS », in UItrasonic and Advanced Methods for Nondestructive Testing and Material Characterization, WORLD SCIENTIFIC, 2007, p. 325-348.

[4] X. P. Maldague, « Introduction to NDT by active infrared thermography », Mater. Eval., vol. 60, nº 9, p. 1060-1073, 2002.

[5] U. Netzelmann et G. Walle, « Induction thermography as a tool for reliable detection of surface defects in forged components », in 17th World Conference on Nondestructive Testing, Shanghai, China, Oct, 2008, p. 25-28.

[6] P. BOUTEILLE, G. LEGROS, H. WALASZEK, « Comparison between Induction Thermography and Conventional NDT Methods for Forged Parts ».

[7] P. Bouteille, G. Legros, S. Maillard, J. Cadith, J. L. Bodnar, « Induction active thermography as an alternative to magnetic particle inspection », in 11th international conference on Quantitative Infrared Thermography, 2012.

[8] B. Oswald-Tranta, « Time-dependent evaluation of inductive pulse heating measurements ».

[9] Maldague X. P. 2001, Theory and Practice of Infrared Technology forNondestructive Testing, John Wiley \& Sons, N. Y.

[10] A. Vageswar, K. K. Balasubramaniam, and C. V. Krishnamurthy, " Wall thinning defect estimation using pulsed IR thermography in transmission mode" Nondestructive Testing and . Eval. 25(4), 333-340 (2010).

[11] C. Ibarra Castanedo. Quantitative Subsurface Defect Evaluation by Pulsed Phase Thermography: Depth Retrieval with the Phase. PhD thesis, University Laval, 2005. 118, 120

[12] Y.He, M. Pan, F. Luo «Defect characterisation based on heat diffusion using induction thermography testing" AIP, Revew of Scientific Instruments, 2012

[13] H. S. Carslaw and J. C. Jaeger, Conduction of Heat in Solids (Oxford University Press, NY, 1959).

[14] F. de Jesus L Rodriguez "Detection and Characterization of Subsurface Defects by Infrared Pulsed Thermography" PhD thesis, Federal University of Santa Catarina 2013 